\title{
Vibrational resonance in an oscillator with an asymmetrical deformable potential
}

\author{
U. E. Vincent, ${ }^{1,2, *}$ T. O. Roy-Layinde, ${ }^{3,4}$ O. O. Popoola,${ }^{4}$ P. O. Adesina, ${ }^{4}$ and P. V. E. McClintock ${ }^{1}$ \\ ${ }^{1}$ Department of Physics, Lancaster University, Lancaster LA1 $4 Y B$, United Kingdom \\ ${ }^{2}$ Department of Physical Sciences, Redeemer's University, Ede, Nigeria \\ ${ }^{3}$ Department of Physics, Olabisi Onabanjo University, Ago-Iwoye, Nigeria \\ ${ }^{4}$ Department of Physics, University of Ibadan, Ibadan, Nigeria
}

(Dated: May 31, 2019)

\begin{abstract}
We report the occurrence of vibrational resonance (VR) for a particle placed in a nonlinear asymmetrical Remoissenet-Peyrard potential substrate whose shape is subjected to deformation. We focus on the possible influence of deformation on the occurrence of vibrational resonance (VR) and show evidence of deformation-induced double resonances. By an approximate method involving direct separation of the timescales, we derive the equation of slow motion and obtain the response amplitude. We validate the theoretical results by numerical simulation. Besides revealing the existence of deformation-induced VR, our results show that the parameters of the deformed potential have a significant effect on the VR and can be employed to either suppress or modulate the resonance peaks, thereby controlling the resonances. By exploring the time series, the phase space structures, and the bifurcation of the attractors in Poincaré section, we demonstrate that there are two distinct dynamical mechanisms that can give rise to deformation-induced resonances, viz: (i) monotonic increase in the size of a periodic orbit; and (ii) bifurcation from a periodic to a quasiperiodic attractor.
\end{abstract}

PACS numbers: 05.40.-a 05.45.-a 87.19.ln

\section{INTRODUCTION}

In physics, resonance traditionally refers to a matching of frequencies giving rise to the amplification of a system's response. Recently, however, resonance has been used more broadly to describe situations where the adjustment of a parameter that is not necessarily a frequency optimises the amplification or response of a system [1]. In the phenomenon of stochastic resonance [2], for instance, it is the noise intensity that is the parameter in question and only in special cases [3] is there also any matching of frequencies. Recent research has proven that many different kinds of external force can also induce resonances and that the latter can manifest in diverse forms, such as chaotic resonance [4-6], stochastic resonance $[2,7]$, coherence resonance $[8,9]$, ghost resonance [10], parametric resonance [1], vibrational resonance [11], anti-resonance [12] and autoresonance [1].

Here we focus on a resonance phenomenon, vibrational resonance (VR), first identified and demonstrated numerically by Landa and McClintock [11] and confirmed theoretically by Gitterman [13] and by Blekhman and Landa $[14,15]$. VR occurs in bi-harmonically driven non-linear systems when there is a large difference between the frequencies of the two driving forces - one of which is a fast oscillation at frequency much higher than that of the slower "signal". In VR, the noise in the stochastic resonance case $[2,7,16-18]$ or chaotic system for chaotic resonance [4-6] is replaced by a high-frequency input leading, for appropriately chosen parameters, to an

*Corresponding author:u.vincent@lancaster.ac.uk enhancement in the system's response to the slow signal. Such amplification takes place when the response amplitude becomes minimum at the bifurcation of the effective potential. Following the foundational studies on VR $[11,13,15]$, vibrational resonance has attracted a lot of research attention and has been reported in bistable systems $[11,15,19]$, multistable systems [20-22], excitable systems [23], ratchets [24], quintic oscillators [25], overdamped systems $[11,21,24]$, coupled oscillators [21, 26], delayed systems [21, 26-28], asymmetric Duffing oscillators [29], fractional order damped oscillators [30-32], feedback networks [33], neuron models [23, 34, 35], a synthetic gene network [36], biological nonlinear maps [37], and systems with nonlinear dissipation [38-40], as well as in harmonically trapped potential systems [41]. In addition, experimental evidence for VR has been reported in bistable and multistable vertical-cavity surface-emitting lasers (VCSELs) [19, 22, 42, 43].

The wide incidence of VR embodied in the above results is intriguing and stimulating, but we note that all of these examples relate to systems that are assumed to have rigid potentials. There are many real systems, however, for which this will not be a good approximation because their potentials undergo deformation - manifested, for example, through variations of crystalline structure, or due to shape distortion, or conformational changes as found in, for instance, a liquid drop, freely suspended in another fluid under the influence of an electric field [4446]. In shape-deformable systems, considerable deviation in the shape of the nonlinear on-site potential from the local potential exist and have for long been used in the context of solitary waves $[47,48]$. Remoissenet and Peyrard $[47,48]$ proposed deformable models of nonlinear systems with deformable periodic substrate potential $V_{R P}(x, r)$, where the parameter, $r$ is the deformable po- 
tential parameter which determines the shape of the potential. The potential $V_{R P}(x, r)$, now often known as the Remoissenet-Peyrard-potential, plays a significant role in one-dimensional atomic chains and has become a subject of diverse research focus [49-61]. Earlier studies, for instance, Nana, et al. [49] used Melnikov theory to predict the onset of chaotic behaviour of a particle in an asymmetric doubly-periodic potential. Similarly, Djuidje et al. [50], observed periodic stick-slip, erratic and intermittent motions, characterized by forced fluctuations, and sliding phenomena for all values of the shape parameter in the range $|r|<1$. Huang et al. [51, 52] reported that directed transport of Brownian particles moving in one- and two-dimensional asymmetric deformable potentials in the presence of asymmetric unbiased fluctuations depends strongly on the potential deformation. Mali et al. [53] observed the appearance of large subharmonic Shapiro steps due to deviations from the sinusoidal potential. More recently, stochastic resonance [54], Farey sequences and Shapiro steps [55], anomalous transport and diffusion phenomena [56-58], current reversals [59], and jump diffusion [60], as well as Devil's staircases [61], have all been investigated. In general, the dynamical and statistical properties are strongly dependent on the variation of the biharmonic parameter, the shape parameter and the phase-lag.

In this paper we investigate the phenomenon of vibrational resonance for a deformable substrate potential, both theoretically and numerically, and we attempt to elucidate the mechanism through which it occurs. More specifically, we will examine the dynamics of a biharmonically driven particle moving in an appropriately nonlinear asymmetrical deformable potential (ASDP) of the kind considered by Remoissenet and Peyrard [48] whose shape is subjected to continuous variation. The rest of the paper is organized as follows. In Sec. II, we present a detailed description of the model under consideration. In Sec. III, a theoretical analysis of VR is presented. In Sec. IV, our numerical results are discussed and compared with the theoretical results. The paper is summarized and conclusions drawn in Sec. V.

\section{MODEL AND ITS DYNAMICS}

We consider the motion of a bi-harmonically driven single particle moving deterministically in an asymmetric potential and modeled by the dynamical equation,

$$
\ddot{x}=-\lambda \dot{x}-\frac{d V_{R P}(x, r)}{d x}+f \cos (\omega t)+g \cos (\Omega t),
$$

where $V_{R P}(x, r)$ is the ASDP and $\lambda$ is a constant damping parameter. The two frequency periodic functions $f \cos (\omega t)+g \cos (\Omega t)$ denotes the bi-harmonic forces with $f$ and $g$ being the amplitudes of the slow and fast driving signals, respectively. The frequencies of the slow driving signal $\omega$ and fast driving signal $\Omega$ are such that $\Omega \gg \omega$.
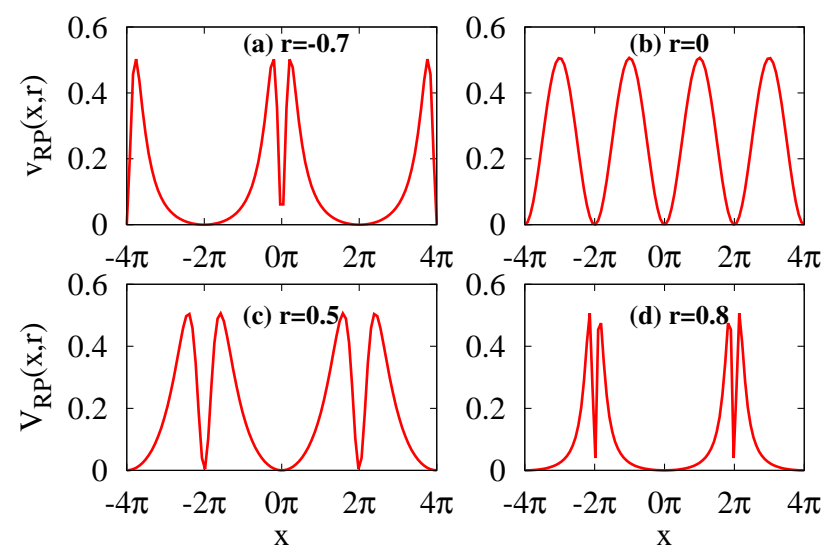

FIG. 1: (Colour online) The asymmetric deformable potential Eq. (2) for different values of $r$ and $V_{0}=10$.

Also, in the analysis of VR it is assumed that $f, g>0$ and can differ by orders of magnitude.

The on-site dimensionless potential $V_{R P}(x, r)$ is given [48] by

$$
V_{R P}(x, r)=\frac{V_{0}}{(2 \pi)^{2}} \frac{\left(1-r^{2}\right)^{2}(1-\cos (2 \pi x))}{\left(1+r^{2}+2 r \cos (\pi x)\right)^{2}},
$$

where $r$ is the deformation potential parameter satisfying $-1<r<1$ and $V_{0}$ is the potential height. For a constant barrier height $V_{0}=10, V_{R P}(x, r)$ reduces to the standard sinusoidal (symmetrical) Sine-Gordon potential when $r=0$, as shown in Fig. 1(b). For other values of $r$ within its allowed range, various deformed and asymmetrical potential shapes can be obtained as shown in Fig. $1(\mathrm{a}, \mathrm{c}, \mathrm{d})$. We note that the potential barriers are of the same height but can have two, inequivalent, sequential, either flat or sharp bottom wells. This implies that the model admits two energetically-equivalent ground states with physically-inequivalent dynamical properties. The flat and sharp bottom wells, i.e. the local minima are located at $x=2 \pi k, k=0, \pm 1, \pm 2, \ldots$, while the local maxima are located at $x=(2 k+1) \pi \pm 2 \cos ^{-1}\left(\frac{2 r}{1+r^{2}}\right), k=$ $0, \pm 1, \pm 2, \ldots$

\section{THEORETICAL ANALYSIS OF VIBRATIONAL RESONANCE}

It is easy to show that Eq. (1) can be expressed as

$$
\begin{aligned}
\ddot{x}=-\lambda \dot{x} & -\frac{V_{0}\left(1-r^{2}\right)^{2}}{\pi} \frac{\sin (\pi x)\left[2 r+\left(1+r^{2}\right) \cos (\pi x)\right]}{\left[1+r^{2}+2 r \cos (\pi x)\right]^{3}} \\
& +f \cos (\omega t)+g \cos (\Omega t),
\end{aligned}
$$

Because the system (3) is subject to two periodic forces, $f \cos (\omega t)$ and $g \cos (\Omega t)$ with $\Omega \gg \omega$, its motion consists of a combination of slow motion $\chi(t)$ at frequency $\omega$ combined with faster motion $\psi(t, \tau=\Omega t)$ at frequency $\Omega$. 
Thus, we may employ here the method of direct separation of the dynamics, to obtain a set of integro-differential equations, one of which describes the slow motion of the system whose response can be modulated by varying the parameters of the high-frequency input drive. By solving the equation for the slow motion, the response amplitude $Q$, defined as the ratio of the amplitude $A$ to the frequency $f$, can be obtained. Thus, the solution $x(t)$ of system (3) can be considered as a superposition of only the solutions $\chi(t)$ of slow evolution with frequency $\omega$ plus that of the fast oscillations $\psi(t)$ at frequency $\Omega$ with $\Omega \gg \omega$, in the form

$$
x(t)=\chi(t)+\psi(t, \Omega t) ;
$$

where we assume that $\chi(t)$ is periodic with period $T=\frac{2 \pi}{\omega}$ and $\psi$ is periodic in the fast time $\tau=\Omega t$ with period $2 \pi$ with its mean value with respect to fast time $\tau$ given by

$$
\langle\psi\rangle=\frac{1}{2 \pi} \int_{0}^{2 \pi} \psi d \tau=0 .
$$

For consistency of analysis, we set all the trigonometric functions in the denominator of $\frac{d V}{d x}$ in Eq. (3) to be of order unity. By making the substitutions

$$
C_{1}=\frac{V_{0}\left(1-r^{2}\right)^{2}}{\pi}, C_{2}=\left(1+r^{2}\right), C_{3}=2 r
$$

in Eq. (3), we have

$$
\begin{aligned}
& \ddot{x}+\lambda \dot{x}+ \frac{C_{1} \sin \pi x\left[C_{3}+C_{2} \cos \pi x\right]}{\left(C_{2}+C_{3} \cos \pi x\right)^{3}} \\
&=f \cos \omega t+g \cos \Omega t .
\end{aligned}
$$

Expanding the denominator in Eq. (7) gives

$$
\ddot{x}+\lambda \dot{x}+x^{U} / x^{L}=f \cos \omega t+g \cos \Omega t .
$$

where $x^{U}=C_{1} \sin \pi x\left[C_{3}+C_{2} \cos \pi x\right]$ and $x^{L}=\left(C_{2}^{3}+\right.$ $\left.3 C_{2}^{2} C_{3} \cos \pi x+3 C_{2} C_{3}^{2} \cos ^{2} \pi x+C_{3}^{3} \cos ^{3} \pi x\right)$. Employing the following trigonometric identities: $\sin \theta \cos \theta=$ $\frac{1}{2} \sin 2 \theta, \cos ^{2} \theta=\frac{1}{2}(\cos 2 \theta+1), \cos ^{3} \theta=\frac{1}{4}(\cos 3 \theta+$ $3 \cos \theta)$ together with the definitions: $\gamma_{1}=C_{1} C_{3}, \gamma_{2}=$ $\frac{C_{1} C_{2}}{2}, \gamma_{3}=\left(C_{2}^{3}+\frac{3 C_{2} C_{3}^{2}}{2}\right), \gamma_{4}=3\left(C_{2}^{2} C_{3}+\frac{C_{3}^{3}}{4}\right), \gamma_{5}=$ $\frac{3 C_{2} C_{3}^{2}}{2}, \gamma_{6}=\frac{C_{3}^{3}}{4}$, in Eq. (8) and simplifying, the equation of motion after some factorisation becomes

$$
\begin{aligned}
\ddot{x} & +\lambda \dot{x}+\frac{\gamma_{1} \sin \pi x+\gamma_{2} \sin 2 \pi x}{\gamma_{3}+\gamma_{4} \cos \pi x+\gamma_{5} \cos 2 \pi x+\gamma_{6} \cos 3 \pi x} \\
& =f \cos \omega t+g \cos \Omega t .
\end{aligned}
$$

Eq. (9) is the simplified form of Eq. (3) which is to be solved. We insert Eq. (4) into Eq. (3), using the expansions

$$
\begin{aligned}
& \sin \pi x=\sin \pi \chi \cos \pi \psi+\sin \pi \psi \cos \pi \chi \\
& \sin 2 \pi x=\sin 2 \pi \chi \cos 2 \pi \psi+\sin 2 \pi \psi \cos 2 \pi \chi \\
& \cos \pi x=\cos \pi \chi \cos \pi \psi-\sin \pi \psi \sin \pi \chi \\
& \cos 2 \pi x=\cos 2 \pi \chi \cos 2 \pi \psi-\sin 2 \pi \psi \sin 2 \pi \chi \\
& \cos 3 \pi x=\cos 3 \pi \chi \cos 3 \pi \psi-\sin 3 \pi \psi \sin 3 \pi \chi .
\end{aligned}
$$

We average both sides with respect to the fast time by using Eq. (5) and the mean values

$$
\begin{aligned}
\langle g \cos \Omega t\rangle & =0, \\
\langle\sin n \pi \psi\rangle & =\frac{1}{2 \pi} \int_{0}^{2 \pi} \sin n \pi \psi d \tau=0, \\
\langle\cos n \pi \psi\rangle & =\frac{1}{2 \pi} \int_{0}^{2 \pi} \cos n \pi \psi d \tau=J_{0}\left(n \pi \psi_{0}\right) .
\end{aligned}
$$

Here, $J_{0}\left(\psi_{0}\right)$ is the zeroth-order Bessel function of the first kind with $\psi_{0}$ being the amplitude of $\psi$, such that

$$
\begin{aligned}
& \sin \pi \chi\langle\cos \pi \psi\rangle+\langle\sin \pi \psi\rangle \cos \pi \chi=J_{0}\left(\pi \psi_{0}\right) \sin \pi \chi \\
& \sin 2 \pi \chi\langle\cos 2 \pi \psi\rangle+\langle\sin 2 \pi \psi\rangle \cos 2 \pi \chi=J_{0}\left(2 \pi \psi_{0}\right) \sin 2 \pi \chi \\
& \cos \pi \chi \cos \pi \psi-\sin \pi \chi\langle\sin \pi \psi\rangle=J_{0}\left(\pi \psi_{0}\right) \cos \pi \chi \\
& \cos 2 \pi \chi \cos 2 \pi \psi-\sin 2 \pi \chi\langle\sin 2 \pi \psi\rangle=J_{0}\left(2 \pi \psi_{0}\right) \cos 2 \pi \chi \\
& \cos 3 \pi \chi \cos 3 \pi \psi-\sin 3 \pi \chi\langle\sin 3 \pi \psi\rangle=J_{0}\left(3 \pi \psi_{0}\right) \cos 3 \pi \chi .
\end{aligned}
$$

We can then write the equation of slow motion as

$$
\ddot{\chi}+\lambda \dot{\chi}+\chi_{1}^{U} / \chi_{1}^{L}=f \cos \omega t,
$$

where $\chi_{1}^{U}=\gamma_{1} J_{0}\left(\pi \psi_{0}\right) \sin \pi \chi+\gamma_{2} J_{0}\left(2 \pi \psi_{0}\right) \sin 2 \pi \chi$ and $\chi_{1}^{L}=\gamma_{3}+\gamma_{4} J_{0}\left(\pi \psi_{0}\right) \cos \pi \chi+\gamma_{5} J_{0}\left(2 \pi \psi_{0}\right) \cos 2 \pi \chi+$ $\gamma_{6} J_{0}\left(3 \pi \psi_{0}\right) \cos 3 \pi \chi$. Eq. (13) is the first of the set of coupled equations for the variable $\chi$. The second equation, for the fast motion $\psi$, is then obtained by subtracting Eq. (7) from Eq. (9). By using the inertial approximation $\ddot{\psi} \gg \dot{\psi} \gg \psi$, the equation of fast motion $\psi$ can then be approximated as that of a damped and periodicallydriven and rapidly oscillating particle whose long-term solution is periodic in fast time $\tau=\Omega t$ as:

$$
\ddot{\psi}+\gamma_{0} \dot{\psi}=g \cos \Omega t,
$$

which has a steady state solution

$$
\psi=\psi_{0} \cos (\Omega t+\theta)=\frac{g}{\Omega \sqrt{\Omega^{2}+\gamma^{2} 0}} \cos (\Omega t+\theta) ;
$$

where

$$
\sin \theta=\frac{-\gamma_{0}}{\sqrt{\Omega^{2}+\gamma^{2} 0}}, \quad \cos \theta=\frac{-\Omega}{\sqrt{\Omega^{2}+\gamma^{2}{ }_{0}}} .
$$

$\psi_{0}$ may be approximated by assuming $\Omega \gg \gamma_{0}$, so that

$$
\psi_{0}=\frac{g}{\Omega^{2}}
$$

whence

$$
\ddot{\chi}+\lambda \dot{\chi}+\chi^{U} / \chi^{L}=f \cos \omega t .
$$

where $\chi^{U}=\gamma_{1} J_{0}\left(\frac{\pi g}{\Omega^{2}}\right) \sin \pi \chi+\gamma_{2} J_{0}\left(\frac{2 \pi g}{\Omega^{2}}\right) \sin 2 \pi \chi$ and $\chi^{L}=\gamma_{3}+\gamma_{4} J_{0}\left(\frac{\pi g}{\Omega^{2}}\right) \cos \pi \chi+\gamma_{5} J_{0}\left(\frac{2 \pi g}{\Omega^{2}}\right) \cos 2 \pi \chi+$ $\gamma_{6} J_{0}\left(\frac{3 \pi g}{\Omega^{2}}\right) \cos 3 \pi \chi$. Eq. (18) is the equation of slow oscillation with the parameters of the fast signal embedded, thus providing the tools for weak signal modulation at 

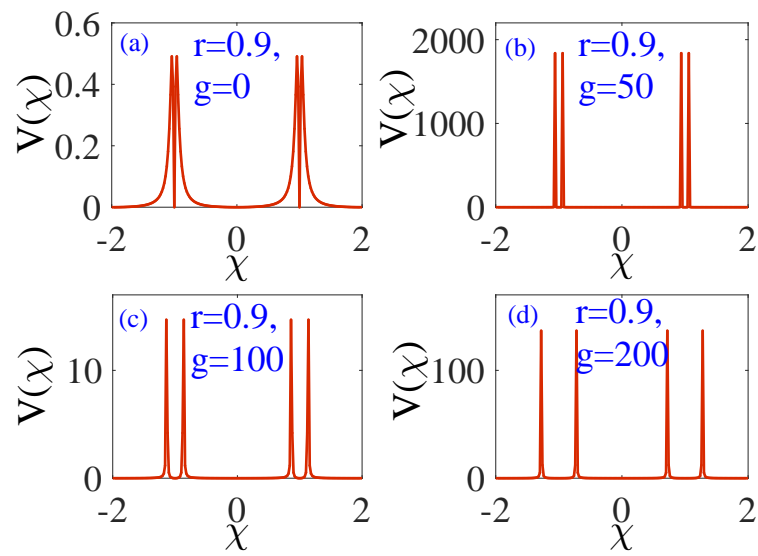

FIG. 2: (Color Online) Effective potential of the deformed system (20) for $r=0.9$ and for four different values of the amplitude, $g=0,50,100$ and 200 in (a)-(d) respectively. $\Omega=$ $20 \omega$ and $\omega=1.5$

the lower frequency through the parameters of the fast signal, i.e. for VR. The effective potential of the system obviously depends on the parameters $\Omega$ and $g$ and can be obtained from

$$
\frac{d V_{\mathrm{eff}}}{d \chi}=V_{\mathrm{eff}}^{U} / V_{\mathrm{eff}}^{L}
$$

where $V_{\text {eff }}^{U}=\gamma_{1} J_{0}\left(\frac{\pi g}{\Omega^{2}}\right) \sin \pi \chi+\gamma_{2} J_{0}\left(\frac{2 \pi g}{\Omega^{2}}\right) \sin 2 \pi \chi$ and $V_{\mathrm{eff}}^{L}=\gamma_{3}+\gamma_{4} J_{0}\left(\frac{\pi g}{\Omega^{2}}\right) \cos \pi \chi+\gamma_{5} J_{0}\left(\frac{2 \pi g}{\Omega^{2}}\right) \cos 2 \pi \chi+$ $\gamma_{6} J_{0}\left(\frac{3 \pi g}{\Omega^{2}}\right) \cos 3 \pi \chi$. Expressing $J_{0_{n}}=J_{0}\left(\frac{n \pi g}{\Omega^{2}}\right)$, with $n=1,2,3$, the effective potential then becomes

$$
V_{\text {eff }}(\chi)=\frac{J_{0_{1}} V_{0}\left(1-r^{2}\right)^{2}}{J_{0_{3}}(2 \pi)^{2}} \frac{(1-\cos 2 \pi \chi)}{\frac{J_{0_{2}}}{J_{0_{1}}}\left(1+r^{2}+2 r \cos \pi \chi\right)^{2}} .
$$

Fig. 2 shows a typical plot of this effective potential Eq. (20) at $r=0.9$ and $\omega=1.5$ for four values of the amplitude of fast input signal, $g=0,50,100$ and 200 in the panels (a)-(d), respectively. It is evident that, for $g=0$, the effective potential shown in Fig. 2(a) is the same as the system potential shown in Fig. 1(d), as would be expected from Eq. (20). Comparison of all panels in Fig. 2 shows that as the amplitude $g$ is increased from 0 to 200, the separation between mirrored/adjacent peaks of the system effective potential increases with amplitude. Moreover, the heights of $V_{\text {eff }}(\chi)$ for the parameters $r=0.9, g=50$ are significantly higher than the other pairs, as shown in Fig. 2(b). To give further insight into the structure of the effective potential, Fig. 3 shows the effect of the parameters of the fast input signal on the $V_{\text {eff }}(\chi)$ for different values of $\Omega$. In Fig. 3(a), the nondeformed potential equivalent of the Sine-Gordon potential (i.e. $r=0$ ), is displayed for four values of amplitude $g(=0,50,100$, and 150$)$ at fixed $\Omega=30$ with lines labelled as 1, 2, 3 and 4 respectively. Whereas the shapes of the peaks remain the same with increase of $g$, the
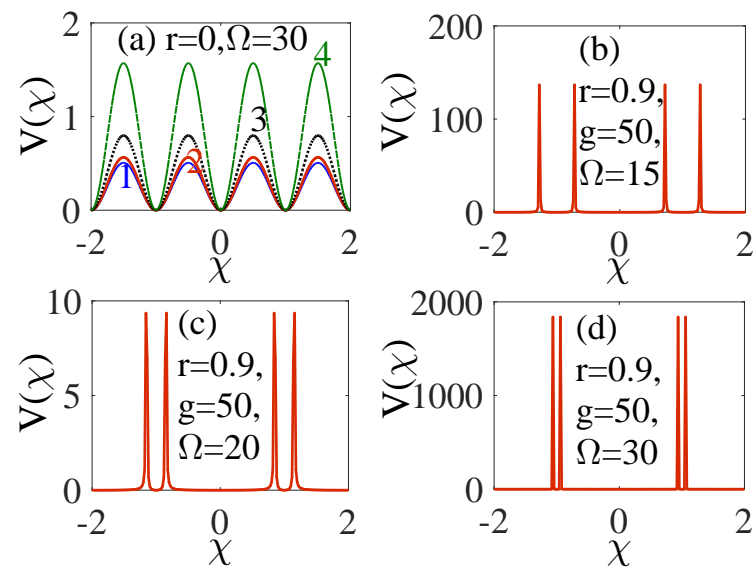

FIG. 3: (Color Online) Effective potential of the deformed system (20) for different parameters. (a) At $r=0, \Omega=30$ for four values of amplitude $g(=0,50,100$, and 150) with line labels $1,2,3$ and 4 respectively. In (b)-(d), $r=0.9, g=0$ but $\Omega=15, \Omega=20$ and $\Omega=30$ respectively.

wells are significantly depressed. In Fig. 3(b)-(d), the deformed effective potential is plotted for fixed values of $r=0.9$ and amplitude $g=50$ and three different frequencies, $\Omega=15, \Omega=20$ and $\Omega=30$. Evidently, increased variations in the fast signal frequency $\Omega$ produce a reversed effect on the $V_{\text {eff }}(\chi)$ as expected from the relation (17).

Next, we describe the system's vibrations in terms of the deviation of slow motion $\chi$ from the equilibrium points $\chi^{*}$, using the deviation variable $Y\left(=\chi-\chi^{*}\right)$ in Eq. (18). The equilibrium points around which slow oscillation can occur are $\chi_{\min (\max )}^{*}=2 n \pi$, where $n$ is an integer. Thus, we can write

$$
\ddot{Y}+\lambda \dot{Y}+V_{J_{0}}^{u} / V_{J_{0}}^{L}=f \cos \omega t,
$$

where $V_{J_{0}}^{u}=\gamma_{1} J_{0_{1}}\left(\sin \pi Y \cos \pi \chi^{*}+\sin \pi \chi^{*} \cos \pi Y\right)+$ $\gamma_{2} J_{0_{2}}\left(\sin 2 \pi Y \cos 2 \pi \chi^{*}+\gamma_{3} \sin 2 \pi \chi^{*} \cos 2 \pi Y\right)$; $V_{J_{0}}^{L}=\gamma_{3}+\gamma_{4} J_{0_{1}}\left(\cos \pi Y \cos \pi \chi^{*}-\sin \pi Y \sin \pi \chi^{*}\right)+$ $\gamma_{5} J_{0_{2}}\left(\cos 2 \pi Y \cos 2 \pi \chi^{*}-\sin 2 \pi Y \sin 2 \pi \chi^{*}\right)+J_{\gamma_{6}}$ and

$$
J_{\gamma_{6}}=\gamma_{6} J_{0_{3}}\left(\cos 3 \pi Y \cos 3 \pi \chi^{*}-\sin 3 \pi Y \sin 3 \pi \chi^{*}\right) .
$$

Using the equilibrium points $\chi^{*}=\chi_{\min (\max )}$, and $J_{0_{n}} \cos n \pi \chi^{*}=\left|J_{0_{n}}\right|$, Eq. (21) can then be written as

$$
\ddot{Y}+\lambda \dot{Y}+Y_{U} / Y_{L}=f \cos \omega t,
$$

where $Y_{U}=\gamma_{1}\left|J_{0_{1}}\right| \sin \pi Y+\gamma_{2}\left|J_{0_{2}}\right| \sin 2 \pi Y$ and ${ }_{L} \gamma_{3}+$ $\gamma_{4}\left|J_{0_{1}}\right| \cos \pi Y+\gamma_{5}\left|J_{0_{2}}\right| \cos 2 \pi Y+\gamma_{6}\left|J_{0_{3}}\right| \cos 3 \pi Y$. Now, using the approximation $f \ll 1,|Y| \ll 1$, sin $n \pi Y=n \pi Y$ and $\cos n \pi Y=1$, Eq. (22) can then be written as a linearly damped-driven oscillator,

$$
\ddot{Y}+\lambda \dot{Y}+\omega_{r}^{2} Y=f \cos \omega t
$$

where $\omega_{r}^{2}=\frac{\gamma_{1}\left|J_{0_{1}}\right|+2 \gamma_{2}\left|J_{0_{2}}\right|}{\gamma_{3}+\gamma_{4}\left|J_{0_{1}}\right|+\gamma_{5}\left|J_{0_{2}}\right|+\gamma_{6}\left|J_{0_{3}}\right|} \pi$. The steady state solution $Y(t)$ of Eq. (23) which describes the ultimate 
behaviour of the system in the long-time limit $t \rightarrow \infty$ is $Y(t)=A_{L} \cos (\omega t+\Phi)$, and the resonant frequency is given as $\omega_{r}=\sqrt{\frac{\gamma_{1}\left|J_{0_{1}}\right|+2 \gamma_{2}\left|J_{0_{2}}\right|}{\gamma_{3}+\gamma_{4}\left|J_{0_{1}}\right|+\gamma_{5}\left|J_{0_{2}}\right|+\gamma_{6}\left|J_{0_{3}}\right|} \pi}$. The system's response amplitude $Q_{\text {analytical which is defined as }}$ the ratio between the output and the forcing signals can be computed from Eq. (23) as;

$$
Q_{\text {analytical }}=\frac{A_{L}}{f}=\frac{1}{\sqrt{\left(\omega_{r}^{2}-\omega^{2}\right)^{2}+\lambda^{2} \omega^{2}}} .
$$

The analytically computed response, $Q_{\text {analytical }}$ is then compared with corresponding numerical computation, $Q_{\text {numerical }}$ which is obtained by solving the actual equation of the system (i.e. Eq. (3)) directly from computational methods in Sec. IV on response curves.

\section{NUMERICAL RESULTS AND DISCUSSIONS}

Our main objective here is to validate the theoretical analysis presented in Sec. III by solving for the response amplitude at frequency $\omega$, which provides an idea of how the low frequency signal is amplified by the high frequency signal, thereby characterizing VR. Furthermore, the implication of VR in the deformable substrate are discussed. First, we express Eq. (3) as coupled firstorder autonomous ordinary differential equations (ODEs) of the form:

$$
\begin{aligned}
\frac{d x}{d t}= & y \\
\frac{d y}{d t}= & -\lambda \dot{x}+f \cos \omega t+g \cos \Omega t \\
& -\frac{V_{0}\left(1-r^{2}\right)^{2}}{\pi} \frac{\sin \pi x\left[2 r+\left(1+r^{2}\right) \cos \pi x\right]}{\left(1+r^{2}+2 r \cos \pi x\right)^{3}} .
\end{aligned}
$$

Next, numerical integration of Eq. (25) was performed using the fourth-order Runge-Kutta scheme with step size $\Delta t=0.01 T$ over a simulation time interval $T_{s}=n T$; $T=\frac{2 \pi}{\omega}$ being the period of the oscillation where $\omega$ is the low frequency input signal and $n(=1,2,3, \ldots)$ is the number of complete oscillations. We used zero initial conditions, a relaxation time of $20 T$ and fixed the values of the potential amplitude and drive parameters at $V_{0}=10$, $f=0.1, \lambda=0.5$ and $\Omega=20 \omega$. These choices ensure that only periodic or quasiperiodic motion is admissible. The other system parameters, $\omega, g, r$ are chosen within a regime so as to optimize VR for $n=100$. For instance, we illustrate in Fig. 4(a) a bifurcation diagram obtained by calculating the time-asymptotic motion of system (25), and visualizing the trajectories as a series of points in $(x, y)$ phase-space, where $y=\dot{x}$ is the particle's velocity; and recording only one point per period of the external drive, i.e. a point is plotted when $\omega t=\delta+n 2 \pi$ where $\delta$ is the Poincaré phase. In Fig. $4(\mathrm{a}), y=\dot{x}$ is plotted as a function of $f$. For increasing values of $f$, the periodic orbit dominates the dynamics in the low frequency

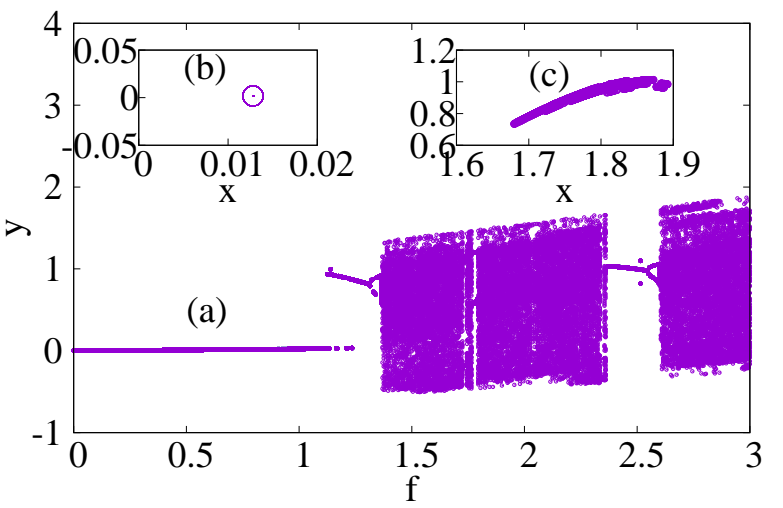

FIG. 4: (Color Online) (a) Bifurcation of the velocity $d x / d t$, as a function of the forcing strength $f$ with fixed parameters $\lambda=0.5$, and $\omega=1.5, \Omega=20 \omega, r=0$, and $g=0$; (b) periodic attractor for $f=0.1$ and (c) chaotic attractor in the Poincaré section $f=1.5$.

regime: $0<f<1.37$ from which the value of $f$ was chosen. For larger values of $f$, small periodic windows are sandwiched by wider chaotic regimes. Fig. 4(b) and (c) illustrates two Poincaré plots showing periodic and chaotic orbits, respectively.

The numerically obtained $Q$-factors are compared with the corresponding analytical values by superposing the response curves for a range of system parameters. The response amplitude $Q$ at the frequency $\omega$ is computed from the Fourier spectrum of the output signal, where $Q_{s}$ and $Q_{c}$ are

$$
\begin{aligned}
& Q_{s}=\frac{2}{n T} \int_{0}^{n T} x(t) \sin \omega t d t \\
& Q_{c}=\frac{2}{n T} \int_{0}^{n T} x(t) \cos \omega t d t .
\end{aligned}
$$

Conventionally, the amplitude is given by,

$$
A=\sqrt{Q_{s}^{2}+Q_{c}^{2}}
$$

and the phase shift as,

$$
\Phi=\tan ^{-1}\left(\frac{Q_{s}}{Q_{c}}\right) .
$$

The response amplitude is thus given as

$$
Q_{\text {numerical }}=\frac{\sqrt{Q_{s}^{2}+Q_{c}^{2}}}{f} .
$$

We begin our examination of the phenomenon of resonance in the system by first considering the case of the non-deformed potential, $r=0$ presented in Fig. 5 for both the theoretical $Q$ ( $\left.Q_{\text {analytical }}\right)$ computed from Eq. (24) and the numerical $Q\left(Q_{\text {numerical }}\right)$ obtained from Eq. (29). Fig. 5(a)-(d) shows the superimposed response 

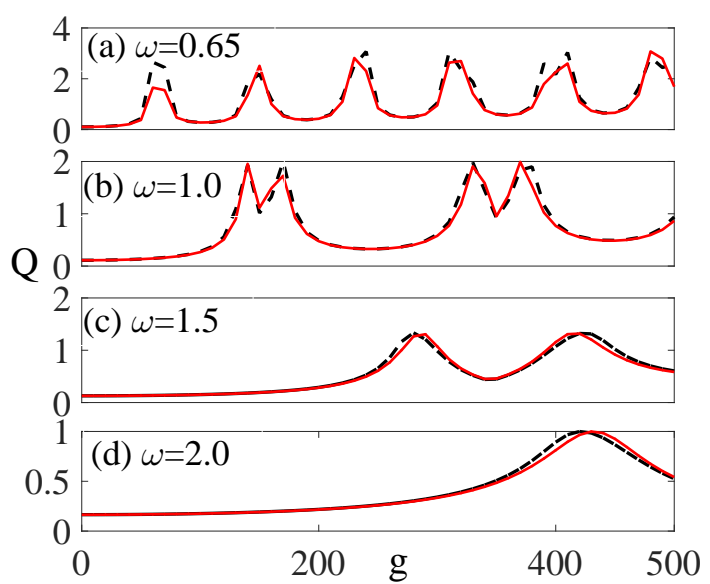

FIG. 5: (Color Online) Dependence of the response amplitude $Q$ on the amplitude $g$ of the high-frequency signal for $r=0, \lambda=0.5, f=0.1$, and $\Omega=20 \omega$, for different values of $\omega(=0.65,0.1,1.5,2)$. The continuous lines represent numerically computed $Q$-values from Eq. (29) while the broken lines represent the corresponding analytical values computed from Eq. (24).

curves depicting the dependence of response amplitude $Q$ on the amplitude $g$ of the high-frequency signal for four values of $\omega(=0.65,0.1,1.5,2)$ respectively. The continuous lines are the response curves of numerically computed $Q$-values while the broken lines represent corresponding analytical values. The other parameter values are $\lambda=0.5, f=0.1$, and $\Omega=20 \omega$. The system is driven into a resonant state by varying the amplitude of the fast signal and the number of peaks is dictated by the lower frequency of the input signal. Here, we see that the VR effect can be induced in the system even in the absence of potential deformation with increasing slow frequency $\omega$. Moreover, there is close agreement between the theoretical and numerical results.

To examine the effect of deformation on the system's response, we show the dependence of the response amplitude $Q$ on the amplitude $g$ of the high-frequency signal for five values of the deformation parameter, $r(=0$, 0.005, 0.007, 0.1, 0.2) in Fig. 6(a)-(e). Here, the choice of $\omega=1.5$ enables us to reproduce Fig. 5(c) as Fig. 6(a) to ensure that the effect of deformation on the system is appropriately observed. The continuous lines represent numerically computed $Q$-values from Eq. (29) while the broken lines represent the corresponding analytical values computed from Eq. (24). The effect of the deformation potential parameter $r$ on the response curves is obvious. As $r$ is gradually increased from zero (as presented in Fig. 6(a)) through low values $r=0.05$ and $r=0.07$ shown in Fig. 6(b) and (b) respectively, there is slight change in the shape of the system's response particularly around the two peaks, but the VR peaks are not significantly affected. The system's response becomes significantly altered at higher values of $r(=0.1$ and 0.2$)$ as shown in Fig. 6(d) and (e), respectively. The effect of

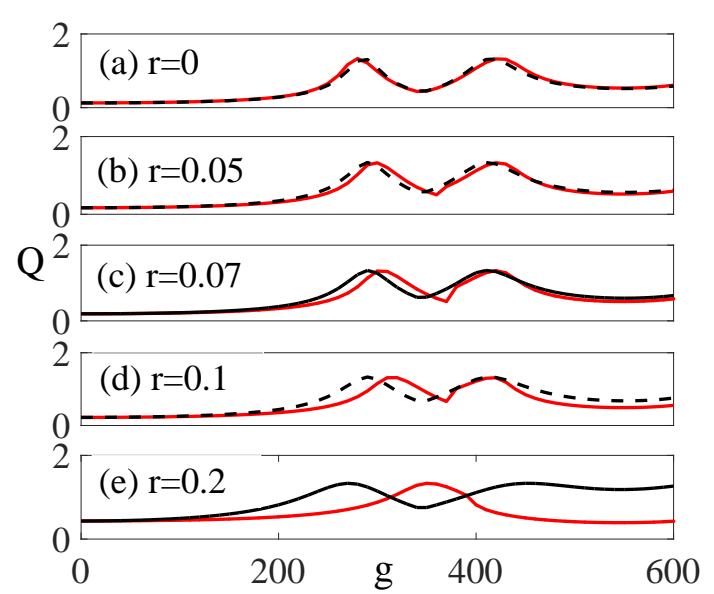

FIG. 6: (Color Online) Dependence of the response amplitude $Q$ on the amplitude $g$ of the high-frequency signal for $\lambda=0.5, f=0.1$, and $\Omega=20 \omega$ and $\omega=1.5$ for five values of $r(=0,0.005,0.007,0.1,0.2)$. The continuous lines represent numerically computed $Q$-values from Eq. (29) while the broken lines represent corresponding analytical values computed from Eq. (24).

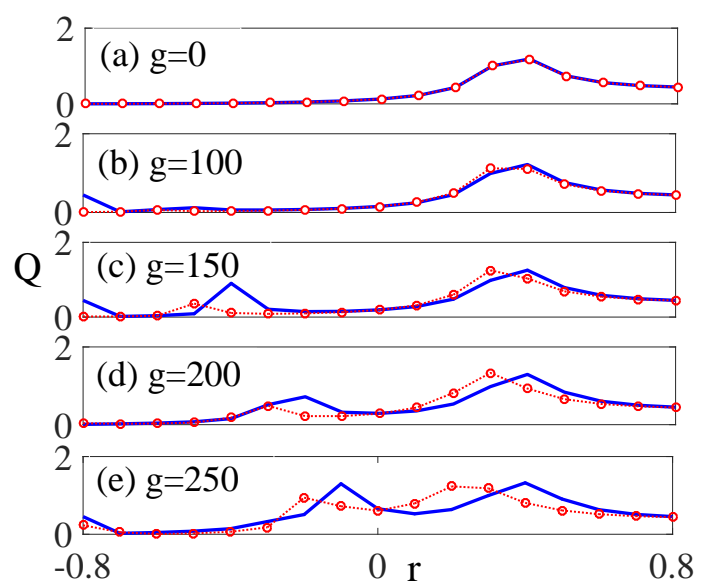

FIG. 7: (Color Online) Dependence of response amplitude $Q$ on the deformation parameter $r$ for $\lambda=0.5, f=0.1$, and $\Omega=20 \omega$ and $\omega=1.5$ for five values of $g(=0,100,150,200$ and $250)$ in Figs. 7(a)-(e) respectively. The continuous lines represent numerically computed $Q$-values form Eq. (29) while the broken lines represent corresponding analytical values computed from Eq. (24).

$r$ on $Q$ becomes well pronounced at $r=0.2$ for which a single peak appears as Fig. 6(e) in contrast with the double peaks observed for in Fig. 6(a)-(d) $r(=0,0.005,0.007$ and 0.1 . This implies that $r$ can be used to modulate the system's response in the resonant state. Comparison between the theoretically and numerically computed $Q$ shows good agreement, with negligible deviation between $Q_{\text {analytical }}$ and $Q_{\text {numerical }}$. However, at larger values of $r$, the deviation becomes quite significant which could intuitively be ascribed to the approximations leading to the linearized equation of motion given by Eq. (23). 
In the preceding discussions, we showed clearly that the VR phenomenon can occur in both the non-deformed and deformed systems. We now proceed to verify the existence of deformation-induced resonances in the presence of bi-harmonic driving signals. Fig. 7 shows the dependence of response amplitude $Q$ on the deformation parameter $r$ for five values of $g(=0,100,150,200$ and 250 ) and fixed frequency, $\omega=1.5$ in panels (a)-(e) respectively. The continuous lines represent numerically computed $Q$-values from Eq. (29) while the broken lines represent the corresponding analytical values computed from Eq. (24). Obviously, the system's response presents a single peak for $g=0$ corresponding to the natural resonance and oscillates in this state for $g \leq 100$. Notice that in Fig. 7(a) the peak only appears at large values of $r$, typically, $r>0$, implying that this is a case of deformation-assisted resonant activation. However, at $g \geq 150$, double resonances are observed as shown in Fig. 7(c)-(e) - the second resonance peaks being VR induced by the high-frequency harmonic driving. In general, as the value of the amplitude $g$ increases from $g=0$ through $g=150$ giving rise to single resonance peak to $g=250$, a second peak and more pronounced peak begins to emerge with increasing values of $g$. Notably, at $g=150$, the second peak is fully formed (See Fig. 7(c)) and two well-pronounced double resonant peaks become fully developed at $g=250$. Zooming on Fig. 7, we illustrate two remarkable effects of turning-on the highfrequency periodic forcing (i.e. $g>0$ ) that are clearly evident: (i) strong enhanced vibration-induced resonances and weak modulation/enhancement of natural resonance. While the peaks of the former are significantly modulated (See Fig. 8(a)), the peaks of the latter are weakly enhanced (Fig. 8(b)). In addition, appreciable agreement between the numerical response factors $Q_{\text {numerical }}$ and the analytical response factors $Q_{\text {analytical }}$ are obtained at lower values of $g$.

Fig. 9 shows the response $Q$ as functions of both the deformed potential parameter $r$ and the amplitude $g$ of the fast signal in the range $(r, g) \epsilon[(-0.8,0.8),(0.0,600)]$, with $\omega=1.5$ and other parameters fixed as before. The red areas in Fig. 9 are regimes of strong resonance, while the regimes with no enhancement are plotted in blue. Elsewhere, however, weak resonance peaks and slight elevations also appear and are indicated with other colours sandwiching from blue and red in the colour bar. The plot reveals regimes in which a good choice of the pair $(r, g)$ leads to either single (see regimes where only red spans across the range of $r$ values) or double-resonance peaks (seen in portions of the plot where two red colors are sandwiched by blue colours). For instance, only one well-pronounced peak is seen at $g=100$ while double peaks are seen at $g=250$ for the range $-0.8 \leq r \leq 0.8$; which is consistent with the analysis presented in Fig. 6 and 7 where both enhancement and control are achievable by means of careful selection of system parameters.

We now turn to explain the underlying dynamics associated with the occurrence of resonances. Here, it is
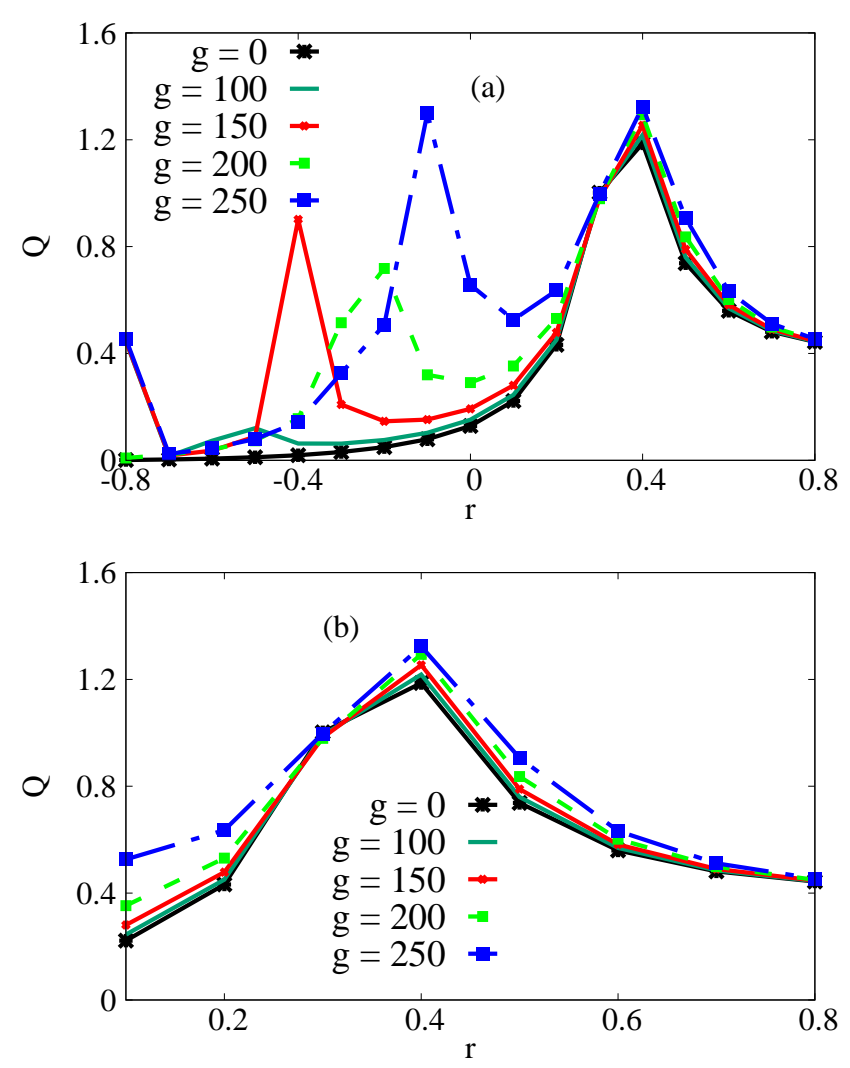

FIG. 8: (Color Online) Dependence of response amplitude $Q$ on the deformation parameter $r$ for $\lambda=0.5, f=0.1$, and $\Omega=20 \omega$ and $\omega=1.5$ for five values of $g(=0,100,150,200$ and 250 ) in Figs. 7(a)-(e) respectively showing in (a) vibrationinduced resonance amplification and (b) weak modulation of primary resonances by VR.

very convenient to employ the time series and phase space plots, as well as the bifurcation of the attractors in Poincaré section. Two distinct mechanisms that could give rise to resonances in the bi-harmonically driven oscillator moving in a deformable potential have been identified: (i) monotonic increase in the size of a periodic attractor; and (ii) bifurcation from a periodic to a quasiperiodic attractor of larger period. In the first case which is concerned with the occurrence of the system's primary resonance, the amplitude of the displacement of a particle is modulated without bifurcation. The system parameters is such that $g=0$, for a fixed value of $r$, with modulation of the oscillation amplitude by varying the frequency $\omega$ of the slow signal input, or $g=0$, for a fixed value of $\omega$ and modulation of the oscillation amplitude by varying the parameter of the potential deformation, i.e. $r$. We illustrate this case in Figure 11. Plotted in Figs. 11(a) and (b) are the time series and phase portraits for $\lambda=0.5, f=0.1$, and $\Omega=20 \omega, g=0$ and $r=0.7$ and for $\omega=0.65,1.5,5.0$. The time series in Fig. 11(a) clearly shows that the particle's displacement is modulated as $\omega$ decreases and in particular, for 


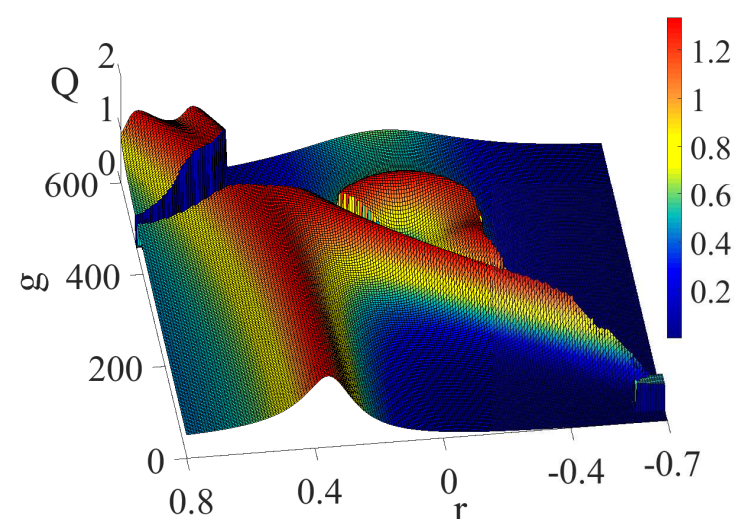

FIG. 9: [Color Online] 3-dimensional plot showing the dependence of the response amplitude $Q$ on the fast signal amplitude $g$ and on the deformation parameter $r$ for $\lambda=0.5$, $f=0.1$, and $\Omega=20 \omega$ and $\omega=1.5$.

$\omega=0.65, A_{0.65} \approx 6.5 A_{1.5}$, where $A_{0.65}$ and $A_{1.5}$ are the displacement amplitudes for $\omega=0.65$ and $\omega=1.5$, respectively. The corresponding phase portraits shown in Fig. 11(b) confirm the monotonic increase in the size of the period 1 orbit. As another example of this scenario, we illustrate in Figs. 11(c) and (d) the effect of amplitude modulation for $g=0$. Here, the magnitude of the deformation parameter $r$ is increased from 0 to 0.7 and correspondingly, the amplitude of the particle's displacement is modulated as depicted by the time series in Fig. 11(c), and a monotonic increase in the size of the period 1 orbit is shown in the phase portrait in Fig. 11(d). The occurrence of resonance for $g=0$ may be understood by considering the deformed effective potential $V_{\text {eff }}(\chi)$ given by Eq. (20) as a combination of regular and deformed potential substrates:

$$
V_{\mathrm{eff}}(\chi)=V_{1}(\chi, r)-k_{1}\left[1+r_{0} \cos (\pi \chi)\right]^{-2} \cos (2 \pi \chi),
$$

where $V_{1}(\chi, r)=\frac{k_{0}}{\frac{J_{02}}{J_{01}}\left[1+r_{0} \cos (\pi \chi)\right]^{2}}$ is the regular part while the second term on the right, $V_{d}=$ $k_{1}\left[1+r_{0} \cos (\pi \chi)\right]^{-2} \cos (2 \pi \chi)$ is the deformed part, $k_{0}=$ $V_{0} \frac{J_{0_{1}}}{J_{0_{3}}} \frac{\left(1-r^{2}\right)^{2}}{(2 \pi)^{2}}$ and $k_{1}=\frac{J_{0_{2}}}{J_{0_{1}}} k_{0}$ is the amplitude of the parametrically modulated periodic force, modulated by $r_{0} \cos (\pi x), r_{0}=r(r+2)$ being the amplitude of modulation. In the absence of the fast signal $g \cos \Omega t$ in Eq. (18), i.e. when $g=0$, the parametrically modulated periodic force plays the role of a complementary harmonic force. Thus, by varying the deformation parameter, $r$, the system's could be driven into its primary resonant state for appropriately chosen parameters.

The second dynamical mechanism leading to resonances, in this case VR, explains the vibration-induced resonances and is connected to a bifurcation from a periodic to a quasiperiodic attractor of larger period. Two typical scenarios for this case are illustrated in Fig. 12. In Fig. 12(a) and (b), the parameters are fixed as before while the values of $g$ are increased from $g=0$ to $g=100$
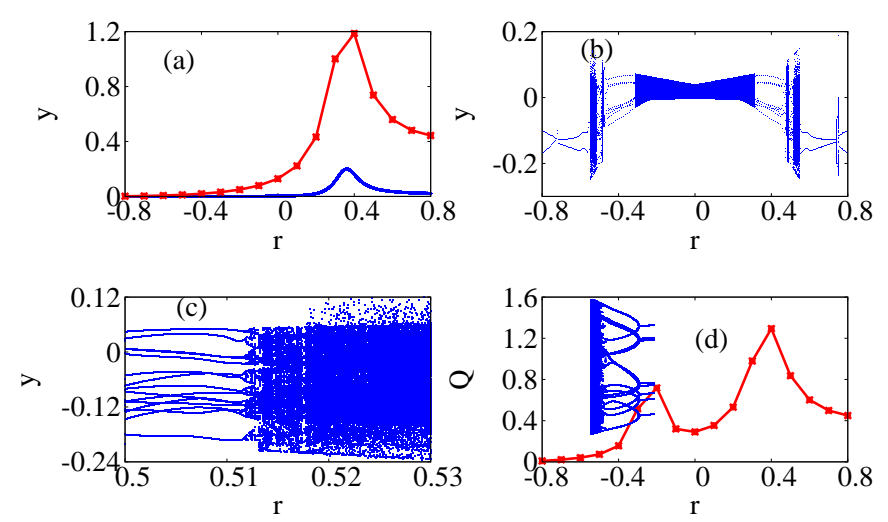

FIG. 10: (Color Online) Local bifurcation structures of the velocity, $y$ (blue dots) and the response amplitude (red lines) as functions of the deformation parameter, $r$ for (a) $g=0$ and (b) $g=200$. Other parameters are fixed as follows: $\omega=1.5$, $\lambda=0.5, \Omega=20 \omega$, and $f=0.1$. (c) Zoom of the bifurcation region $0.5 \leq r \leq 0.53$ in (b). (d) Response amplitude, $Q$ for $g=200$ corresponding to (b). The inset in (d) is the zoom of the multiple reversed period-doubling bifurcation region $-0.48 \leq r \leq-0.45$.

for a non-deformed system (i.e. $r=0$ ). For $r=0, g=0$, the particle moves periodically with a small amplitude, $A_{0} \approx 0.025$. When $g$ is increased from 0 to 100 , the motion of the particle becomes quasiperiodic with amplitude modulation. The corresponding phase portrait depicts the creation of a higher-order quasiperiodic orbit at $g=50$. Similarly, the periodic-quasiperiodic orbit bifurcation can also take place $\forall r>0$ as shown in Fig. 12(c) and (d). In this latter case, however, the deformation complements the role of the fast signal amplitude $g$ in the occurrence of VR, such that when $r>0, g>0$, higher amplitude VR is obtained. Notably the amplitude modulation shown in Fig. 12(c) and (d) can also be achieved by fixing the value of $g$ while varying $r$.

To complete the discussions, we present in Fig. 10 the bifurcation diagrams (blue dots) and the response amplitude (red lines) for $g=0$ and $g=200$ both as functions of the deformation parameter $r$, while other system parameters are fixed. In the range $-0.8<r<0$, when the high-frequency periodic forcing is turned-off (i.e. $g=0$ ) shown in Fig. 10(a), the strength of the deformation is insufficient to trigger any motion, confining the particle to a quiescent state with zero constant velocity. For $r \geq 0$ the particle moves rapidly from the quiescent state with increasing velocity, attaining its peak at $r \approx 0.36$. Consequently, the system resonates with the size of the periodic orbit increasing monotonically as shown in Fig. 11 without qualitative change in the system's dynamics. This resonant state for $g=0$ is not vibration-induced, but rather is the system's primary resonance due to resonance oscillation induced by the deformation parameter, $r$. On the other hand, when $g=50>0$ as illustrated in Fig. 10(b) there is clear evidence of qualitative 

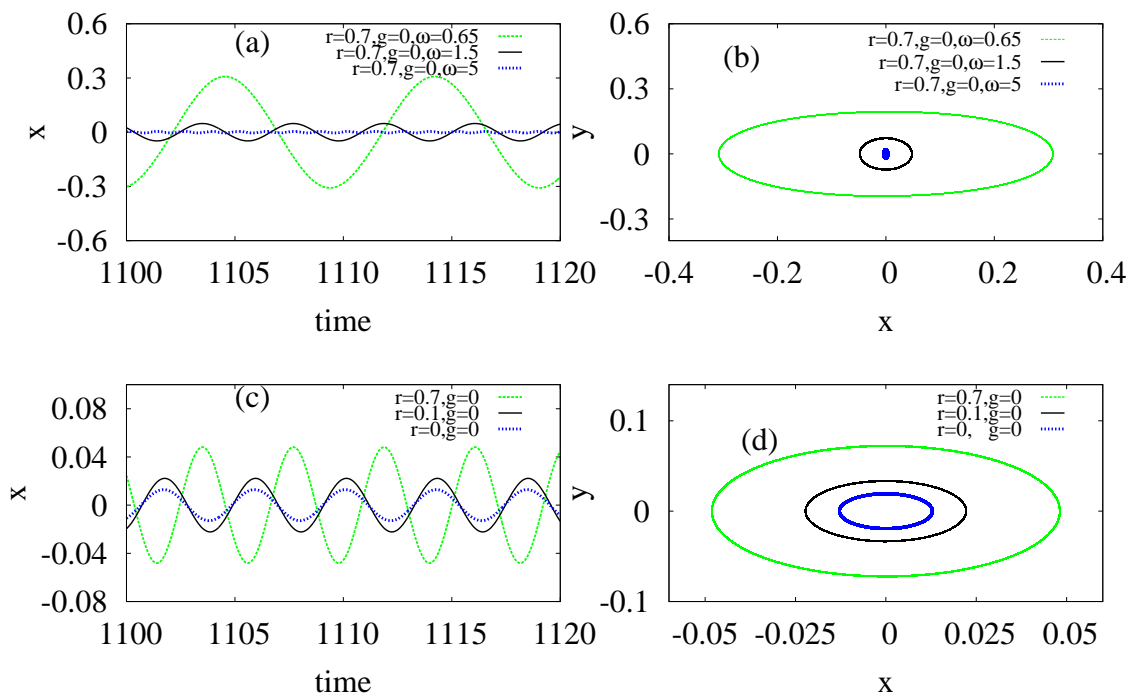

FIG. 11: (Color Online) Time series and phase portraits for $\lambda=0.5, f=0.1$, and $\Omega=20 \omega$ and $g=0$. (a) and (b) $r=0.7$ and for increasing values of $\omega(=0.65,1.5,5.0)$; (c) and (d) $\omega=1.5$ and for increasing values of $r(=0,0.1,0.6)$.
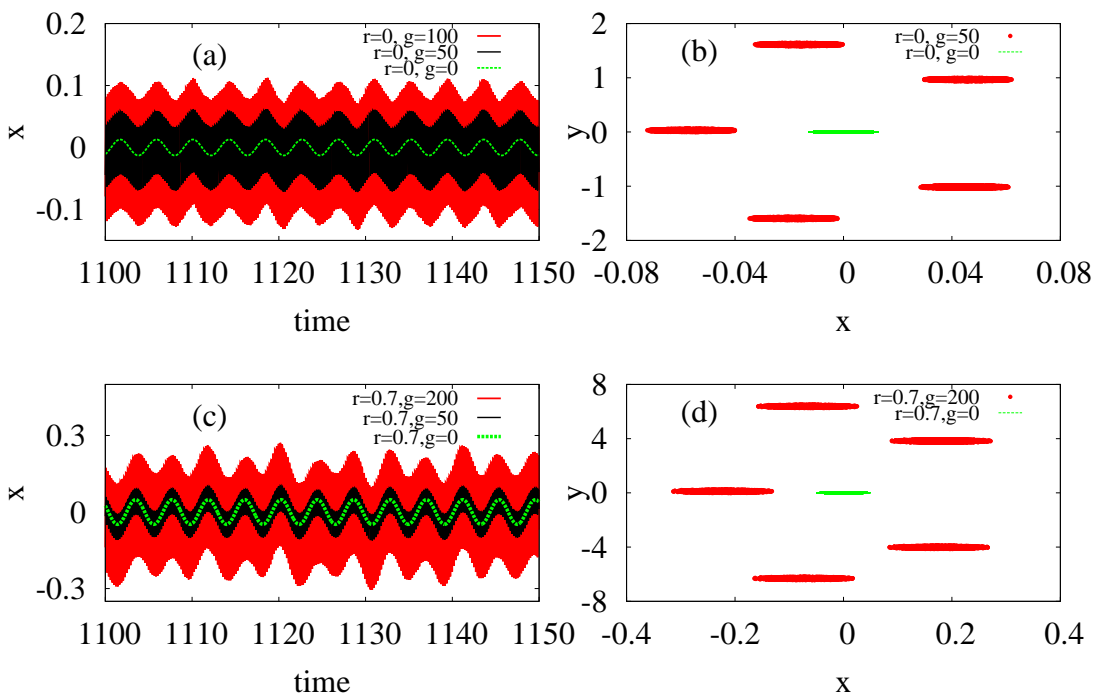

FIG. 12: (Color Online) Time series and phase portraits for $\lambda=0.5, f=0.1$, and $\Omega=20 \omega$ and $\omega=1.5$ and different values of $g(=0,50,100)$. Green dotted lines $(g=0$, periodic orbit), black solid line $(g=50$, quasiperiodic orbit), blue dashed line ( $g=200$, quasiperiodic orbit). (a) and (b) for $r=0$; (c) and (d) for $r=0.7$.

changes in the system's dynamics. The local bifurcation structure shows that the particle's dynamics is predominantly periodic-quasiperiodic bifurcation transitions for $-0.8<r<0.8$. Higher order quasiperiodic orbits up to period 15 in the range $0.5 \leq r \leq 0.513$ and born from Hopf bifurcations in the right branch may appear as shown in the zoomed bifurcation regions of Fig. 10(c); while multiple period-doubling bifurcation in the range $-0.48 \leq r \leq-0.45$ (see Fig. 10(d)) in some $r$-parameter regimes of the left branch may additionally occur (See the inset of Fig. 10(d)).

The occurrence of a second peak (i.e. vibration-induced resonances), giving rise to double resonance as illustrated in Fig. 7 when $g>0$ can be understood by zooming on the parameter regime of the bifurcation diagram in Fig. 10(b) for which the second resonance peak appears as shown in Fig. 10(d). For $r \leq-0.47$ and $g=0$ shown in Fig. 10(d), the particle is confined to a quiescent state with zero constant velocity, so that no resonance is observed. However, as the strength of the deformation increases, such that $r>-0.47$ and $g$ taken non-zero values, the particle optimizes the fast signal's amplitude, $g$ to accelerate via multiple reverse period-doubling/periodadding bifurcations of its orbits thereby leading to VR in 
some $r$-parameter region where $Q$-values were near zero for $g=0$. The $r$-parameter region for which multiple reverse period-doubling occurs is dependent on the values of $g$. Thus, we can affirm that the occurrence of the double-resonances is closely connected to the multiple reversed period-doubling bifurcations.

\section{CONCLUSIONS}

Many real systems exist for which a fixed, regular, potential will not be a good approximation because their potentials undergo deformation as manifested in, for instance, variations of crystalline structure, or conformational changes in a liquid drop freely suspended in another fluid under the influence of an electric field. In such shape-deformable systems, there may be considerable deviation in the shape of the nonlinear on-site potential from the local potential. For example, in material science the deformation may appear as changes in the shape or size of an object due to an applied tensile, compressive, shear, bending or torsion force, or due to a change in temperature. In both crystalline and non-crystalline solids, the mobility of structural defects such as point vacancies, grain boundaries, stacking faults, screw and line dislocations and twins are features that could impact significantly on structural vibrations. Hence detailed investigation of their effects is essential for a wide range of applications of vibrational dynamics. Our investigation of VR for a bi-harmonically driven particle moving in a nonlinear asymmetrical deformable potential has led to some interesting results. The system was similar to that considered by Remoissenet and Peyrard [48]. Its shape was subjected to continuous variation, and we investigated the resultant effects on VR, based on a separa- tion of timescales to derive the equation of slow motion, and an approximation to obtain the response amplitude. Both in the approximate analytic theory and in numerical simulations, we found that the deformation parameters have a significant effect on VR. Furthermore, they can be employed either to suppress, induce or to modulate the resonance peaks, i.e. to control the occurrence of resonances. The appropriate $(r, g)$-parameter space of the system was scanned to locate the $(r, g)$-parameter values for VR and to discover how its control could be achieved. By exploration of the time series, the phase space structures, and the bifurcation of attractors in Poincaré section, the underlying dynamics associated with the occurrence of VR was elucidated. We conclude that, for this system with a deformable potential substrate, there are two distinct dynamical mechanisms which can give rise to resonances, depending on the parameters of the system: (i) a monotonic increase in the size of a periodic orbit; and (ii) bifurcation from a periodic attractor to a quasiperiodic attractor of larger period.

Acknowledgments

UEV is supported by the Royal Society of London, through their Newton International Fellowship Alumni scheme. The research was supported by the Engineering and Physical Sciences Research Council UK (grant No $\mathrm{EP} / \mathrm{M} 015831 / 1)$. No new data were created during this study. The results in Fig. 9 were obtained from a MATLAB Code computed over a period of two months on the High End Computing (HEC) facility at Lancaster University, UK. We thank Dr. Gemma Lancaster for useful discussions. The authors are indeed grateful to the reviewers for their constructive comments.
[1] S. Rajasekar and M. A. F. Sanjuan, Nonlinear Resonances, Springer Series in Synergetics (Springer, Switzerland, 2016).

[2] M. I. Dykman, D. G. Luchinsky, R. Mannella, P. V. E. McClintock, N. D. Stein, and N. G. Stocks, "Stochastic resonance in perspective," Nuovo Cimento D 17, 661-683 (1995).

[3] N. G. Stocks, N. D. Stein, S. M. Soskin, and P. V. E. McClintock, "Zero-dispersion stochastic resonance," J. Phys. A: Math. Gen. 25, L1119-Ll125 (1992).

[4] L. Y. Chew, C. Ting, and C. H. Lai, "Chaotic resonance: Two-state model with chaos-induced escape over potential barrier," Phys. Rev. E 72, 036222 (2005).

[5] Isao T. Tokuda, Cheol E. Han, Kazuyuki Aihara, Mitsuo Kawato, and Nicolas Schweighofer, "The role of chaotic resonance in cerebellar learning," Neur. Net. 23, 836 842 (2010).

[6] Sou Nobukawa, Haruhiko Nishimura, and Teruya Yamanishi, "Chaotic resonance in typical routes to chaos in the Izhikevich neuron model," Sci. Rep. 7, 1331 (2017).

[7] Jesús Casado-Pascual, José Gómez-Ordónez, and
Manuel Morillo, "Stochastic resonance: theory and numerics," Chaos 15, 26115 (2005).

[8] Arkady S. Pikovsky and Jürgen Kurths, "Coherence resonance in a noise-driven excitable system," Phys. Rev. Lett. 78, 775-778 (1997).

[9] O. V. Ushakov, H. J. Wünsche, F. Henneberger, I. A. Khovanov, L. Schimansky-Geier, and M. A. Zaks, "Coherence resonance near a Hopf bifurcation," Phys. Rev. Lett. 95, 123903 (2005).

[10] S. Rajamani, S. Rajasekar, and M. A. F. Sanjuán, "Ghost-vibrational resonance," Comm. Nonlin. Sc. Num. Sim. 19, 4003-4012 (2014).

[11] P. S. Landa and P. V. E. McClintock, "Vibrational resonance," J. Phys. A: Math. Gen. 33, L433 (2000).

[12] R. Jothimurugan, K. Thamilmaran, S. Rajasekar, and M. A. F. Sanjuán, "Multiple resonance and antiresonance in coupled Duffing oscillators," Nonlin. Dyn. 83, 1803-1814 (2016).

[13] M. Gitterman, "Bistable oscillator driven by two periodic fields," J. Phys. A: Math. Gen. 34, L355 (2001).

[14] I. I. Blekhman, Vibrational Mechanics (World Scien- 
tific,Singapore, 2000).

[15] I.I Blekhman and P.S Landa, "Conjugate resonances and bifurcations in nonlinear systems under biharmonical excitation," Intern. J. Non-Lin. Mech. 39, 421 - 426 (2004).

[16] Ruoshi Yuan, Xinan Wang, Yian Ma, Bo Yuan, and Ping Ao, "Exploring a noisy Van der Pol type oscillator with a stochastic approach," Phys. Rev.. E 87, 062109 (2013).

[17] S Saikia, A. M. Jayannavar, and Mangal C Mahato, "Stochastic resonance in periodic potentials," Phys. Rev. E 83, 061121 (2011).

[18] Jing Li, "Enhancement and weakening of stochastic resonance for a coupled system," Chaos 21, 043115 (2011).

[19] V. N. Chizhevsky, "Vibrational higher-order resonances in an overdamped bistable system with biharmonic excitation," Phys. Rev. E 90, 042924 (2014).

[20] S. Rajasekar, K. Abirami, and M. A. F. Sanjuan, "Novel vibrational resonance in multistable systems," Chaos 21, 033106 (2011).

[21] J. H. Yang and X. B. Liu, "Controlling vibrational resonance in a multistable system by time delay," Chaos 20, 033124 (2010).

[22] V. N. Chizhevsky, "Experimental evidence of vibrational resonance in a multistable system," Phys. Rev. E 89, 062914 (2014).

[23] Bin Deng, Jiang Wang, Xi-le Wei, Haitao Yu, and Huiyan Li, "Theoretical analysis of vibrational resonance in a neuron model near a bifurcation point," Phys. Rev. E 89, 062916 (2014).

[24] M. Borromeo and F. Marchesoni, "Vibrational ratchets," Phys. Rev. E 73, 016142 (2006).

[25] S. Jeyakumari, V. Chinnathambi, S. Rajasekar, and M. A. F. Sanjuan, "Analysis of vibrational resonance in a quintic oscillator," Chaos 19, 043128 (2009).

[26] J H Yang and X B Liu, "Controlling vibrational resonance in a delayed multistable system driven by an amplitude-modulated signal," Phys. Scrip. 82, 025006 (2010).

[27] C. Jeevarathinam, S. Rajasekar, and M. A. F. Sanjuan, "Theory and numerics of vibrational resonance in Duffing oscillators with time-delayed feedback," Phys. Rev. E 83, 066205 (2011).

[28] C Jeevarathinam, S Rajasekar, and M Sanjuan, "Effect of multiple time-delay on vibrational resonance," Chaos 23, 013136 (2013).

[29] S. Jeyakumari, V. Chinnathambi, S. Rajasekar, and M. A. F. Sanjuan, "Vibrational resonance in an asymmetric Duffing oscillator," Intern. J. Bifurc. Chaos 21, 275-286 (2011).

[30] J Yang and H Zhu, "Vibrational resonance in Duffing systems with fractional-order damping," Chaos 22, 013112 (2012).

[31] Tianqi Qin, Tianting Xie, Maokang Luo, and Ke Deng, "Vibrational resonance in fractional-order overdamped multistable systems," Chinese J. Phys. 55, 546 - 555 (2017).

[32] T. L. M. Djomo Mbong, M. Siewe Siewe, and C. Tchawoua, "The effect of the fractional derivative order on vibrational resonance in a special fractional quintic oscillator," Mech. Res. Commun. 78, 13-19 (2016).

[33] Ying-mei Qin, Jiang Wang, Cong Men, Bin Deng, and Xi-le Wei, "Vibrational resonance in feedforward network," Chaos 21, 023133 (2011).

[34] B. Deng, J. Wang, X-le. Wei, K. M. Tsang, and W. L. Chan, "Vibrational resonance in neuron populations,"
Chaos 20, 013113 (2010).

[35] H. Yu, J. Wang, C. Liu, B. Deng, and Xi-le Wei, "Vibrational resonance in excitable neuronal systems," Chaos 21, 043101 (2011).

[36] Jiancheng Shi, Chusheng Huang, Tao Dong, and Xiuzhi Zhang, "High-frequency and low-frequency effects on vibrational resonance in a synthetic gene network," Phys. Biol. 7, 036006 (2010).

[37] S. Rajasekar, J. Used, A. Wagemakers, and M. A. F. Sanjuan, "Vibrational resonance in biological nonlinear maps," Commun. Nonlin. Sci. Num. Sim. 17, 3435-3445 (2012).

[38] T. L. M. Djomo Mbong, M. Siewe Siewe, and C. Tchawoua, "The effect of nonlinear damping on vibrational resonance and chaotic behavior of a beam fixed at its two ends and prestressed," Commun. Nonlin. Sci. Num. Sim. 22, 228-243 (2015).

[39] T. O. Roy-Layinde, J. A. Laoye, O. O. Popoola, and U. E. Vincent, "Analysis of vibrational resonance in biharmonically driven plasma," Chaos 26, 093117 (2016).

[40] T. O. Roy-Layinde, J. A. Laoye, O. O. Popoola, U. E. Vincent, and P. V. E. McClintock, "Vibrational resonance in an inhomogeneous medium with periodic dissipation," Phys. Rev. E 96, 032209 (2017).

[41] K. Abirami, S. Rajasekar, and M.A.F. Sanjuan, "Vibrational resonance in a harmonically trapped potential system," Commun. Nonlin. Sci. Numer. Sim. 47, 370-378 (2017).

[42] J. P. Baltanás, L. López, I. I. Blechman, P. S. Landa, A. Zaikin, J. Kurths, and M. A. F. Sanjuán, "Experimental evidence, numerics, and theory of vibrational resonance in bistable systems," Phys. Rev. E 67, 066119 (2003).

[43] V. N. Chizhevsky, "Noise-induced suppression of nonlinear distortions in a bistable system with biharmonic excitation in vibrational resonance," Phys. Rev. E 92, 032902 (2015).

[44] R. S. Allan and S. G. Maso, "Particle behaviour in shear and electric fields i. deformation and burst of fluid drops," Proc. R. Soc. Lond. A 267, 45-61 (1962).

[45] Etienne Lac and G. M. Homsy, "Axisymmetric deformation and stability of a viscous drop in a steady electric field," J. Fluid Mech. 590, 239-264 (2007).

[46] Michael Zabarankin, "Toroidal drop under electric field: arbitrary drop-to-ambient fluid viscosity ratio," Proc. R. Soc. Lond. A 473, 20170379 (2017).

[47] M. Peyrard and M. Remoissenet, "Solitonlike excitations in a one-dimensional atomic chain with a nonlinear deformable substrate potential," Phys. Rev. B 26, 28862899 (1982).

[48] M. Remoissenet and M. Peyrard, "Soliton dynamics in new models with parametrized periodic double-well and asymmetric substrate potentials," Phys. Rev. B 29, 3153-3166 (1984).

[49] L. Nana, T.C. Kofané, E. Coquet, and P. Tchofo-Dinda, "Chaotic behaviour in deformable models: the asymmetric doubly periodic oscillators," Chaos, Solitons \& Fractals 13, $731-740$ (2002).

[50] G. Djuidjé Kenmoé and T. C. Kofané, "Frictional stickslip dynamics in a nonsinusoidal Remoissenet-Peyrard potential," Eur. Phys. J. B 55, 347-354 (2007).

[51] X. Q. Huang, P. Deng, J. W. Xiong, and B. Q. Ai, "Directed transport in deformable ratchets," Eur. Phys. J. B 85, 162 (2012). 
[52] X. Q Huang, Peng Deng, and Bao-Quan Ai, "Transport of Brownian particles in a deformable tube," Physica A 392, 411 - 415 (2013).

[53] Petar Mali, Jasmina Tekić, and Milan Pantić, "Saturation effects in ac+dc driven frenkel-kontorova model," Comm. Nonlin. Sci. Numer. Sim. 19, 3469 - 3475 (2014).

[54] G. Djuidjé-Kenmoé, Y. J. Ngouongo-Wadop, and T. C. Kofané, "Effect of the potential shape on the stochastic resonance processes," J. Stat. Phys. 161, 475-485 (2015).

[55] Jovan Odavić, Petar Mali, and Jasmina Tekić, "Farey sequence in the appearance of subharmonic shapiro steps," Phys. Rev. E 91, 052904 (2015).

[56] Rosalie Laure Woulaché, Fabrice Maxime Kepnang Pebeu, and Timoléon C. Kofané, "Dynamics of Brownian motors in deformable medium," Physica A 460, 326 334 (2016).

[57] André Marie Fopossi Mbemmo, Djuidjé-Germaine Kenmoé, and Timoléon Crépin Kofané, "Anomalous transport and diffusion phenomena induced by bihar- monic forces in deformable potential systems," Eur. Phys. J. B 89, 211 (2016).

[58] A. M. Fopossi Mbemmo, G. Djuidjé Kenmoé, and T. C. Kofané, "Shape potential effects on transport and diffusion phenomena," Fluct. Noise Lett. 16, 1750011 (2017).

[59] Chun-Chun Cai, Jian-Li Liu, Hao Chen, and Feng-Guo $\mathrm{Li}$, "Current reversals of an underdamped Brownian particle in an asymmetric deformable potential," Commun. Theor. Phys. 69, 266-270 (2018).

[60] A. Kotri, E. Elkoraychy, Y. Lachtioui, M. Mazroui, and Y. Boughaleb, "Jump diffusion in the strong-collision model on deformable substrate potential," Opt. Quant. Electron. 48, 101 (2016).

[61] I. Sokolović, P. Mali, J. Odavić, S. Radošević, S. Yu. Medvedeva, A. E. Botha, Yu. M. Shukrinov, and J. Tekić, "Devil's staircase and the absence of chaos in the dc- and ac-driven overdamped Frenkel-Kontorova model," Phys. Rev. E 96, 022210 (2017). 